\title{
New concepts in the treatment of malignant pleural mesothelioma
}

\author{
Moshe Lapidot, Samuel Freyaldenhoven, Raphael Bueno \\ Division of Thoracic Surgery, Lung Center and International Mesothelioma Program, Brigham and Women's Hospital and Harvard Medical School, \\ Boston, Massachusetts, USA \\ Correspondence to: Moshe Lapidot; Raphael Bueno. Division of Thoracic Surgery, Lung Center and International Mesothelioma Program, \\ Brigham and Women's Hospital and Harvard Medical School, 75 Francis Street Boston, Boston, MA 02115, USA. Email: mlapidot@partners.org; \\ rbueno@partners.org. \\ Provenance: This is an invited Editorial commissioned by Section Editor Dr. Wankun Chen (Department of Anesthesiology, Fudan University \\ Shanghai Cancer Center, Shanghai, China). \\ Comment on: Wald O, Sugarbaker DJ. New concepts in the treatment of malignant pleural mesothelioma. Annu Rev Med 2018;69:365-77.
}

Submitted Feb 01, 2018. Accepted for publication Feb 06, 2018.

doi: $10.21037 /$ jtd.2018.02.75

View this article at: http://dx.doi.org/10.21037/jtd.2018.02.75

Malignant pleural mesothelioma (MPM) is an aggressive solid malignancy with a median overall survival of 8 months (1). First line combination therapy of cisplatin and pemetrexed has brought an overall survival benefit of only 11 weeks compared to cisplatin alone $(2,3)$. Despite the limited efficacy of this regimen, it has continued as standard MPM therapy 14 years after its acceptance, underscoring the urgent need for novel treatments that improve the lives of MPM patients. Within this focused review (4), Wald and Sugarbaker elaborate on novel advanced classification and prognostication methods that shine a light on the path to personalized medicine, possibly matching the right therapy with the right patient. Several key principles and evolving concepts in the care of MPM patients are discussed.

As well known in MPM, surgical resection of the tumor is a major therapeutic option that is often combined with chemotherapy. A major controversy in this field has been the appropriate identification of patients that would benefit from multimodality treatments (MMT) protocol. In this review the authors also refer to known biomarkers for that aim. The tumor histology and lymph node status profoundly affect long term survival and treatment strategy for a patient with mesothelioma. As reported in several recent papers $(5,6)$, MPM with pure epithelioid histology is associated with significantly prolonged survival. Consequently, the authors emphasize the importance of a proper and representative tissue sample for avoiding misclassification of mesothelioma subtype even with the means of more invasive procedures. In the recent work of Friedberg and colleagues long term outcomes of 73 MPM patients with histology confirmed epithelioid subtype treated with MMT protocol were demonstrated. The median overall and disease-free survival was 3 and 1.2 years, respectively (7). In addition, the authors discuss the changes in the 2016 MPM staging system which are summarized by unification of the previous $\mathrm{T} 1 \mathrm{a}$ and $\mathrm{T} 1 \mathrm{~b}$ tumors into a new $\mathrm{T} 1$ category and the unification of the previous N2 lymph nodes into the N1 category based on accumulated prognostic data. In the previous staging system patients with $\mathrm{N} 2$ nodes were regarded as stage III with median survival of 14 months while according with the new staging system they belong to stage II group with improvement of 5 months in the median survival. In the paucity of properly established clinical trials comparing chemotherapy to surgery based MMT, we agree with the authors attitude and stress the importance of surgery based MMT protocols for mesothelioma treatment based on retrospective and prospective cohort studies. The international mesothelioma interest group viewpoint as well as the National Comprehensive Cancer Network (NCCN) highlight the important role of surgical macroscopic complete resection (MCR) and control of micro metastatic disease as part of MPM MMT protocols (8). MCR can be achieved by pleurectomy decortication (PD) or by extrapleural pneumonectomy (EPP). Specifically, the most prolonged median survival is achieved in patients with epithelioid histology who have relative small tumor volume and complete the entire MMT protocol. Median survival for this group is in the range of 29-39 months. Given 
the higher mortality and morbidity with EPP the authors rightfully point out that achieving MCR while limiting surgery-associated morbidity and mortality are the prime goal of the surgical component of MMT. Even with the most aggressive MMT protocols, cancer control rates are low. Consequently, researchers continue to explore new treatment avenues.

Over the past years, there has been an increasing growth in our understanding of mesothelioma. New knowledge in mesothelioma genetics, epigenetics, tumor microenvironment and immunobiology. Current phase 2 trials with biological drugs and immune checkpoint inhibitors have shown new promise, but not enough to change therapy standards (9) with the potential exception of the anti-angiogenic agent bevacizumab which reportedly increased survival by 2 months when added to cisplatin and pemetrexed therapy (10). New clinical trials using immunotherapies and genetically engineered $\mathrm{T}$ cells (CAR-T cells) targeting the tumor antigens such as mesothelin and WT1 show encouraging results.

Surgical series repeatedly showed that a subset of $15-30 \%$ of MPM patients treated with combined surgery and chemotherapy enjoys long term survival. In most of the cases these survivals have epithelioid histology and early to intermediate stage disease. However, not all patients with epithelioid histology have a long-term survival and part of the survivals are composed from patients with mixed histology subtype or positive lymph node. Given that, there is a need for novel biomarkers to identify that subset of patients who can benefit from long term survival. As mentions in Wald and Sugarbaker review, in the Nature Genetics paper, our group profiled 216 matched MPM tumor-normal tissue pairs by RNAseq and exome seq (11). We utilized the data to define four robust and distinct more homogeneous expression clusters consistent with the epithelial to mesenchymal transformation and demonstrated that one of these (expression cluster 1) defines the epithelioid tumors with best outcomes. It is important to note that just $38 \%$ of the histologically epithelioid type were defined as genetically epithelioid (expression cluster 1) while the remaining $62 \%(88 / 141)$ of histologically epithelioid samples, classified as biphasic-epithelioid expression ( $\mathrm{n}=51)$, biphasic-sarcomatoid expression $(\mathrm{n}=29)$ or sarcomatoid expression $(\mathrm{n}=8)$ and showed lower overall survival. The sarcomatoid expression cluster contained all eight samples histologically classified as sarcomatoid/ desmoplastic and $21 \%(13 / 62)$ of histologically biphasic samples. The ultimate goal is to better pre-operatively predict and define patients who likely to benefit from MMT based therapy based on more accurate combination of molecular, imaging and other parameters.

\section{Acknowledgements}

None.

\section{Footnote}

Conflicts of Interest: The authors have no conflicts of interest to declare.

\section{References}

1. Beebe-Dimmer JL, Fryzek JP, Yee CL, et al. Mesothelioma in the United States: a Surveillance, Epidemiology, and End Results (SEER)-Medicare investigation of treatment patterns and overall survival. Clin Epidemiol 2016;8:743-50.

2. Krug LM. An overview of chemotherapy for mesothelioma. Hematol Oncol Clin North Am 2005;19:1117-36.

3. Kindler HL. Systemic treatments for mesothelioma: standard and novel. Curr Treat Options Oncol 2008;9:171-9.

4. Wald O, Sugarbaker DJ. New Concepts in the Treatment of Malignant Pleural Mesothelioma. Annu Rev Med 2018;69:365-77.

5. Bölükbas S, Manegold C, Eberlein M, et al. Survival after trimodality therapy for malignant pleural mesothelioma: Radical Pleurectomy, chemotherapy with Cisplatin/ Pemetrexed and radiotherapy. Lung Cancer 2011;71:75-81.

6. Lang-Lazdunski L, Bille A, Papa S, et al. Pleurectomy/ decortication, hyperthermic pleural lavage with povidone-iodine, prophylactic radiotherapy, and systemic chemotherapy in patients with malignant pleural mesothelioma: a 10-year experience. J Thorac Cardiovasc Surg 2015;149:558-65; discussion 565-6.

7. Friedberg JS, Simone CB 2nd, Culligan MJ, et al. Extended Pleurectomy-Decortication-Based Treatment for Advanced Stage Epithelial Mesothelioma Yielding a Median Survival of Nearly Three Years. Ann Thorac Surg 2017;103:912-9.

8. Rusch V, Baldini EH, Bueno R, et al. The role of surgical cytoreduction in the treatment of malignant pleural mesothelioma: meeting summary of the International Mesothelioma Interest Group Congress, September 11-14, 2012, Boston, Mass. J Thorac Cardiovasc Surg 2013;145:909-10.

9. Kindler HL, Karrison TG, Gandara DR, et al. 
Multicenter, double-blind, placebo-controlled, randomized phase II trial of gemcitabine/cisplatin plus bevacizumab or placebo in patients with malignant mesothelioma. J Clin Oncol 2012;30:2509-15.

10. Zalcman G, Mazieres J, Margery J, et al. Bevacizumab for newly diagnosed pleural mesothelioma in the Mesothelioma Avastin Cisplatin Pemetrexed Study

Cite this article as: Lapidot $M$, Freyaldenhoven S, Bueno R. New concepts in the treatment of malignant pleural mesothelioma. J Thorac Dis 2018;10(3):1283-1285. doi: $10.21037 /$ jtd. 2018.02 .75
(MAPS): a randomised, controlled, open-label, phase 3 trial. Lancet 2016;387:1405-14.

11. Bueno R, Stawiski EW, Goldstein LD, et al. Comprehensive genomic analysis of malignant pleural mesothelioma identifies recurrent mutations, gene fusions and splicing alterations. Nat Genet 2016;48:407-16. 